\title{
Prior Therapeutic Experiences, Not Expectation Ratings, Predict Placebo Effects: An Experimental Study in Chronic Pain and Healthy Participants
}

\author{
Luana Colloca $^{\mathrm{a}-\mathrm{c}}$ Titilola Akintola ${ }^{\mathrm{a}, \mathrm{c}}$ Nathaniel R. Haycock ${ }^{\mathrm{a}}$ Maxie Blasini $^{\mathrm{a}}$ \\ Sharon Thomas ${ }^{a} \quad$ Jane Phillips $^{d}$ Nicole Corsi $^{a} \quad$ Lieven A. Schenk $^{\mathrm{a}, \mathrm{c}}$ \\ Yang Wang ${ }^{a, c}$ \\ ${ }^{a}$ Department of Pain and Translational Symptom Science, School of Nursing, University of Maryland, Baltimore, MD, \\ USA; ${ }^{b}$ Departments of Anesthesiology and Psychiatry, School of Medicine, University of Maryland, Baltimore, MD, \\ USA; ' Center to Advance Chronic Pain Research, University of Maryland, Baltimore, MD, USA; d Department of Neural \\ and Pain Sciences, School of Dentistry, University of Maryland, Baltimore, MD, USA
}

\section{Keywords \\ Clinical research - Cognition · Conditioning - Psychology · Psychosomatic medicine}

\begin{abstract}
Introduction: Many clinical trials fail because of placebo responses. Prior therapeutic experiences and patients' expectations may affect the capacity to respond to placebos in chronic disorders. Objective: The scope of this study in 763 chronic orofacial pain and healthy study participants was to compare the magnitude and prevalence of placebo effects and determine the putative role of prior therapeutic experiences vs. expectations. Methods: We tested placebo propensity in a laboratory setting by using 2 distinct levels of individually tailored painful stimulations (high pain and low pain) to reinforce expectations and provide a hypoalgesic experience (conditioning phase). Afterwards, both levels of pain were surreptitiously set at a moderate pain level to test for placebo effects (testing phase). Pain and expectation ratings were assessed as primary outcomes using visual analog scales. Results: In both chronic pain and healthy participants, placebo effects were similar in magnitude, with the larger prevalence of responders in the healthy participants.
\end{abstract}

Although chronic pain participants reported higher pain relief expectations, expectations did not account for the occurrence of placebo effects. Rather, prior experience via conditioning strength mediated placebo effects in both pain and healthy participants. Conclusions: These findings indicate that participants with chronic pain conditions display robust placebo effects that are not mediated by expectations but are instead directly linked to prior therapeutic experiences. This confirms the importance of assessing the therapeutic history while raising questions about the utility of expectation ratings. Future research is needed to enhance prediction of responses to placebos, which will ultimately improve clinical trial designs.

(c) 2020 S. Karger AG, Basel

\section{Introduction}

Placebo effects are reductions in symptoms that result from patients' perceptions of a therapeutic intervention $[1,2]$. They depend on the activation of descending neural pathways [3] and, in some instances, on patients' expectations [4]. These effects can be carefully investigated in laboratory settings and used as a tool to

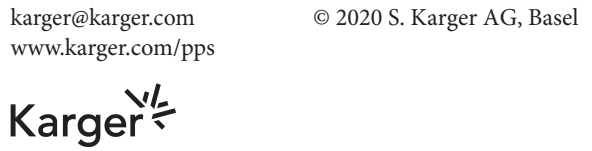

Luana Colloca

Department of Pain and Translational Symptom Science, University of Maryland 655 W Lombard St. Suite 729A

Baltimore, MD 21201 (USA)

colloca@umaryland.edu 
Table 1. Sociodemographic and clinical characteristics of the study participants

\begin{tabular}{|c|c|c|}
\hline Characteristic & $\begin{array}{l}\text { TMD } \\
(n=363)\end{array}$ & $\begin{array}{l}\mathrm{HC} \\
(n=400)\end{array}$ \\
\hline Age, years & $41.41 \pm 0.74$ & $29.42 \pm 0.52$ \\
\hline African-American/Black race & $142(39)$ & $101(25)$ \\
\hline Females sex & $278(77)$ & $238(60)$ \\
\hline College graduate or higher & & \\
\hline educational level ${ }^{\mathrm{a}}$ & $222(61)$ & $294(74)$ \\
\hline $\begin{array}{l}\text { Annual income USD } 60,000 \text { or } \\
\text { higher }\end{array}$ & $154(42)$ & $169(42)$ \\
\hline Blood pressure, $\mathrm{mm} \mathrm{Hg}$ & & \\
\hline Systolic & $127.76 \pm 0.87$ & $119.82 \pm 0.67$ \\
\hline Diastolic & $80.18 \pm 0.51$ & $73.91 \pm 0.49$ \\
\hline Heart rate, bpm & $73.97 \pm 2.62$ & $73.97 \pm 0.49$ \\
\hline BMI & $28.41 \pm 0.38$ & $25.28 \pm 0.26$ \\
\hline \multicolumn{3}{|l|}{ TMD type ${ }^{b}$} \\
\hline Myalgia & $343(95)$ & \\
\hline Myofascial pain with referral & $33(9)$ & \\
\hline Right arthralgia & $265(73)$ & \\
\hline Left arthralgia & $255(70)$ & \\
\hline TMD headache & $187(52)$ & \\
\hline GCPS $(0-4)$ & $2.02 \pm 0.06$ & \\
\hline JFLS (0-10) & $2.06 \pm 0.98$ & \\
\hline $\mathrm{OBC}(0-84)$ & $28.22 \pm 0.68$ & \\
\hline Pain duration, months & $144.28 \pm 7.14$ & \\
\hline \multicolumn{3}{|l|}{ Pain medicine } \\
\hline NSAID & $87(24)$ & \\
\hline Muscle relaxants & $10(3)$ & \\
\hline $\mathrm{BDZ}$ & $17(5)$ & \\
\hline TCA & $5(1)$ & \\
\hline SSRI & $39(11)$ & \\
\hline SNRI & $25(7)$ & \\
\hline SARI & $12(3)$ & \\
\hline NDRI & $16(4)$ & \\
\hline
\end{tabular}

explore the contribution of endogenous descending modulatory systems to pain reduction. We used a pain model to explore how expectations shape placebo effects in healthy controls and in a target population of temporomandibular disorder (TMD), a chronic pain condition that occurs in $5-10 \%$ of the general population [5-10]. Prior studies on placebo effects have been conducted with both behavioral and/or biological approaches in chronic irritable bowel syndrome [11-13], idiopathic and neuropathic pain [14-16], low back pain [17-19], migraine [20], and knee osteoarthritis [21]. Very often, these studies lack comparison with healthy participants, although a recent meta-analysis reported a greater benefit from placebo treatment in patients than in healthy participants [22]. Also, the relative contributions of expectations to the magnitude of placebo effects is variable, and thus further exploration is needed, par-

\begin{tabular}{lcr} 
Characteristic & $\begin{array}{l}\text { TMD } \\
(n=363)\end{array}$ & \multicolumn{1}{l}{$\begin{array}{l}\mathrm{HC} \\
(n=400)\end{array}$} \\
\hline $\begin{array}{l}\text { Other pain disorders } \\
\quad \text { Ehlers-Danlos syndrome }\end{array}$ & $3(1)$ & \\
Knee pain & $42(12)$ & \\
$\quad$ Shoulder pain & $46(13)$ & \\
Low back pain & $118(33)$ & \\
Osteoarthritis & $73(20)$ & \\
$\quad$ Fibromyalgia & $17(5)$ & \\
$\quad$ Migraine & $219(60)$ & \\
$\quad$ Headache & $49(14)$ & \\
Mood disorders & & \\
$\quad$ BDI score & $10.73 \pm 0.54$ & $6.47 \pm 0.32$ \\
$\quad$ STAI score & $39.94 \pm 0.59$ & $35.36 \pm 0.43$ \\
Temperature used, ${ }^{\circ} \mathrm{C}$ & & \\
$\quad$ Conditioning phase & $46.5 \pm 0.11 /$ & $47.49 \pm 0.1 /$ \\
$\quad$ (red/green stimuli) & $40.76 \pm 0.12$ & $41.71 \pm 0.11$ \\
$\quad$ Testing phase & & \\
$\quad$ (red = green stimuli) & $46.07 \pm 0.18$ & $46.24 \pm 0.1$ \\
\hline
\end{tabular}

Values are presented as means \pm SEM or numbers (\%). HC, healthy controls; GCPS, Grades of Chronic Pain Scale; JFLS, Jaw Function Limitation Scale; OBC, Oral Behavior Checklist; NSAID, nonsteroidal anti-inflammatory drugs; BDZ, benzodiazepine; TCA, tricyclic antidepressants; SSRI, selective serotonin reuptake inhibitors; SNRI, selective serotonin-norepinephrine reuptake inhibitor; SARI, serotonin antagonist and reuptake inhibitors; NDRI, norepinephrine-dopamine reuptake inhibitor; BDI, Beck Depression Inventory; STAI, State Trait Anxiety Inventory. ${ }^{\text {a }} \mathrm{Col}-$ lege graduation or higher vs. some college or lower. ${ }^{\mathrm{b}}$ TMD types often overlapped.

ticularly across clinical conditions. Therefore, this experimental study determined the occurrence of, magnitude of, and response to placebo effects comparatively in TMD patients and healthy controls. We addressed the questions: (1) do participants with chronic orofacial pain respond similarly (magnitude and duration) to placebo effects than controls and (2) do prior therapeutic experience and verbally-reported expectations influence such effects?

\section{Materials and Methods}

\section{Patient Population}

A total of 796 potential participants were screened for this study from August 2016 to November 2019 at the University of Maryland (Baltimore, MD, USA). Twenty-one were excluded for not meeting the criteria for eligibility. Of the remaining 775 , three 


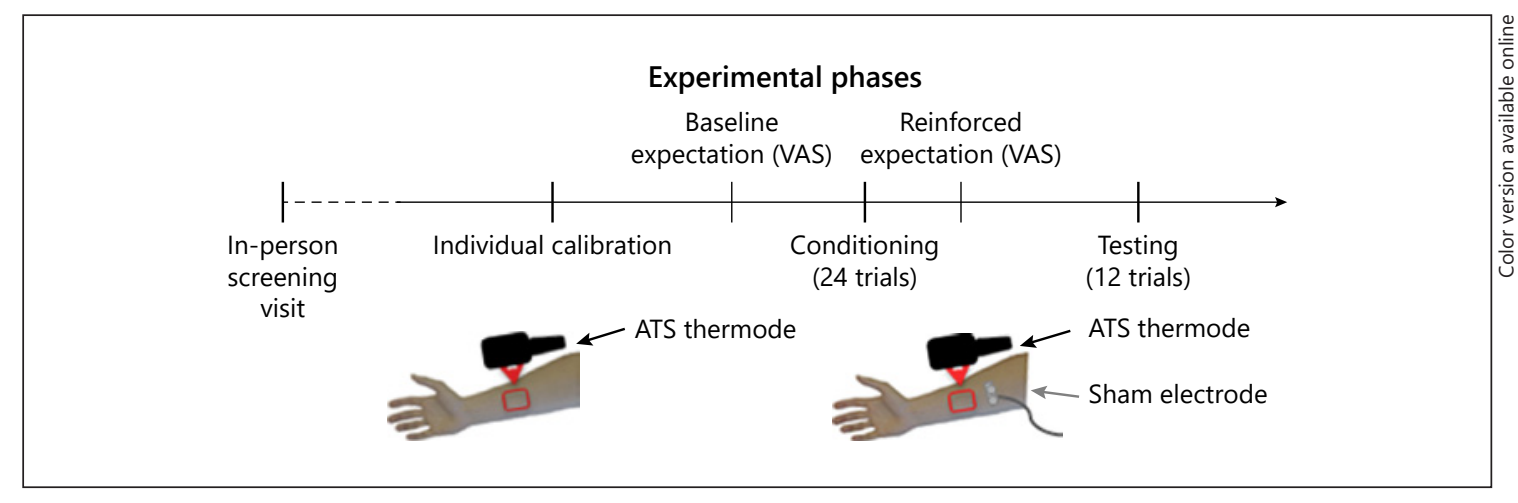

Fig. 1. Experimental paradigm. This study started with an in-person screening visit at the orofacial clinic to confirm the diagnosis of TMD. Once the diagnosis was confirmed for TMD patients and a healthy status was confirmed for control participants, the experimental session took place. After consenting, the individual warmth and pain sensitivity was calibrated (calibration phase). An ATS thermode was used to induce pain via thermal heat stimulations. Warmth and pain sensitivity were assessed to define the intensity of stimuli to induce low, moderate, and high painful experiences to be used during the conditioning and testing phases. An ascending series of stimuli was delivered, starting at a nonpainful threshold, until warm and painful sensations were induced. Initially, thermal heat stimuli at a very low and usually imperceptible level were delivered. Next, the investigator increased the intensity of the stimuli in steps until of the participants reached a threshold, indicated by a level that they felt was "definitely painful, but tolerable." To minimize floor effects by ensuring that this level of stimulation was at least somewhat painful, when this threshold level was reached, the participant was asked to verbally report his or her pain on a numerical rating scale of 1 (minimum pain) to 10 (maximum tolerable pain). If their reported pain was less than 6 , the subject was asked if he or she felt comfortable trying a higher intensity, such that the

who passed the screening were lost to follow-up and 9 discontinued this study, leaving 363 TMD participants and 400 pain-free controls (Table 1).

\section{Inclusion/Exclusion Criteria}

Participants who fulfilled the following criteria were included in this study: age between 18 and 65 years, TMD pain duration longer than 3 months, and meeting the TMD diagnostic criteria [23, 24]. The diagnosis of TMD was confirmed with a clinical examination by an independently trained, calibrated examiner according to the Axis I Diagnostic Criteria for TMD (DC/TMD) [23] at the Brotman Facial Pain Clinic, School of Dentistry, University of Maryland. Clinical pain was also assessed with an Axis II evaluation via the Graded Chronic Pain Scale [25], the Jaw Function Limitation Scale [26], and the Oral Behaviors Checklist for parafunctional behaviors [27]. The control group consisted of participants without pain of any nature, neurological or psychiatric disorders, or medication use.

\section{Placebo Procedure}

Painful thermal stimuli were delivered to the ventral forearm using the Medoc Pathway system (Medoc Advanced Medical Systems, Ramat Yishai, Israel). An experimenter in a white coat ex- participants' pain ratings at the end of calibration were at least 6 out of 10 on the numerical rating scale that was used instead of the VAS to facilitate the experimenter-participant interaction during the calibration. Once the calibration was completed, the participants entered the conditioning phase that provided them with distinct nonpainful and painful experiences that were repetitively measured to obtain an individualized index of conditioning strength. Expectations were rated at baseline prior to any therapeutic experience and after the conditioning phase. Participants' self-reported expectations were assessed before and after the conditioning phase using a $0-100$ VAS scale to address the question: how much do you think this procedure will reduce your pain? Afterwards, the testing phase took place. A sham electrode was attached proximal to the ATS thermode to the dominant arm of the participant. Participants were instructed that when the electrode was activated, the pain experience would be reduced. During the testing phase, both red and green screens were paired with a moderate temperature of heat pain stimuli in order to assess for placebo effects. Differences between red- and green-related ratings was operationally defined as placebo effects. For both the conditioning and testing phases, after each trial the participants were asked to rate the intensity of the pain on a VAS with "no pain" and "maximum pain tolerable" as anchors.

plained that a green color displayed on a computer screen would indicate the delivery of nonpainful thermal stimuli while a red color would indicate tailored painful thermal stimuli. Participants were told that the green-associated stimuli were made less painful by activation of an electrode (actually a sham), which would deliver imperceptible electrical stimuli to reduce the thermal stimuli (Fig. 1a). This was repeated over a 24-trial conditioning phase to expose participants to repeated pain relief (so-called "conditioning strength") to use as a proxy for prior "therapeutic" experiences. Then, unbeknownst to the participant, the intensity of the red-and green-associated stimuli was surreptitiously set at the same, moderate level during a 12-trial testing phase. Any difference in redversus green-associated pain reports was operationally defined as "placebo analgesia" [28-33]. Pain intensities for the conditioning and testing phases were tailored to the individual participants' pain sensitivity as previously reported [30-32, 34]. Verbally reported expectations of pain relief were assessed at the baseline and after the conditioning phase (reinforced expectations).

Outcomes and assessment

Pain intensities during the conditioning and testing phases and expectations were set as primary outcomes. Experimental pain in- 
tensity ratings were assessed using a $100-\mathrm{mm}$ visual analog scale (VAS) anchored from "no pain" to "maximum tolerable pain." The distance from the zero anchor to the middle of the patients' marks was measured and quantified as XX millimeters out of $100 \mathrm{~mm}$ (Celeritas Inc.). Similarly, participants rated the expectation of "How much do you think this procedure will reduce your pain?" using the 100-mm VAS.

\section{Statistical Analyses}

Based on this meta-analysis [22], we assumed a small placebo difference (Cohen's $d=0.25$ ) between TMD and healthy control participants (minimum: $n=254$ for each group for a 0.8 statistical power at an a level of 0.05). ANCOVA for repeated measurement was conducted to test for conditioning strength and placebo effects. Delta scores of each individual participants' pain intensity ratings for red-paired stimuli and green-paired stimuli were calculated for the conditioning phase (12 repeated deltas) and for the testing phase (6-repeated deltas). Group (TMD patients vs. healthy controls) was treated as the between-subjects factor, and the covariables of age, sex, race, educational level, and level of pain used (i.e., temperature of heat pain used in the conditioning/testing phase) were controlled. Where applicable, we used post hoc comparisons with Bonferroni correction and Spearman correlations. We defined the "responder status" by implementing a previously adopted classification of responders versus nonresponders [35], which accounts for trial-by-trial VAS rating variability (see online suppl. material; for all suppl. material, see www.karger.com/ doi/10.1159/000507400).

We measured the effect size of placebo analgesia and compared Cohen's $d$ between cases and controls using a Fisher $r$ - $t$ - $z$ transformation. Given the clinical relevance of these findings, we computed the number needed to treat (NNT) in both case and control cohorts [36]. Finally, we determined the potential mediating role of reinforced expectations in driving placebo effects in TMD and healthy control participants using Hayes's model [37, 38]. An a value of 0.05 was used for all analyses, which were conducted using SPSS version 26 (SPSS Inc., Chicago, IL, USA).

\section{Results}

Overall, the multivariate linear regression with age, sex, race, educational level, annual income, and BMI as predictors revealed that older age $(\beta=-0.11, p=0.005)$ was associated with smaller placebo effects, while a higher level of education $(\beta=0.08, p=0.043)$ was associated with larger effects (sub-cohort analyses are shown in the online suppl. material). TMD pain severity, pain duration, and usage of medication, as well as concurrent presence of depression and anxiety, did not influence placebo analgesia (online suppl. Table S1).

During the conditioning phase controlling for individual levels of given painful temperature, we observed a significant main effect of group on the $12 \delta$ scores of red minus green pain intensity $\left(F_{1,752}=5.79, p=0.016\right)$. TMD participants displayed a lower conditioning strength (mean $=57.39$, SEM $=0.98)$ than control participants (mean $=60.60$, SEM $=0.92$ ), suggesting that TMD patients may have experienced less treatment effectiveness.

When testing for placebo effects, we found a significant main effect of $\delta$ scores during the testing phase (repeated measures ANCOVA, $F_{1,754}=164.05, p<0.001=$ 0.017 ) with significantly lower pain intensity ratings for green trials (mean $=31.78$, SEM $=0.85$ ) versus red trials (mean $=50.24$, SEM $=0.85, p<0.001$ ). We controlled for interrater variability in pain ratings and compared the magnitude of placebo effects in TMD patients and controls. We found a nonsignificant main effect of group (TMD patients vs. controls) on placebo analgesia $\left(F_{1,754}=0.01, p=0.992\right)$, indicating that the magnitude of these effects was comparable in TMD (mean $=18.39$, SEM $=1.03$ ) and control participants (mean $=18.40$, $\mathrm{SEM}=0.97, p=0.992$; Fig. $2 \mathrm{a}$ ). The effect size for placebo effects within TMD patients (Cohen's $d=1.08$ ) was not significantly different from that within controls (Cohen's $d=1.26, Z=1.07$; Fisher $r$ - $t$ - $z$ transformation, $p=0.143$ ). We further determined the extinction pattern of placebo effects for TMD patients and controls and found a similar pattern of duration (main effect of trials, $F_{5,3,770}=0.50$, $p=0.780$ ), indicating that placebo effects did not significantly extinguish in both groups (interaction trials by group, TMD patients vs. controls, $F_{5,3,770}=0.90, p=$ $0.480)$.

In terms of pain relief expectations before and after the conditioning phase, there was a significant main effect of time $\left(F_{1,753}=102.63, p<0.001\right)$, with significantly greater expectations after the conditioning phase (mean = $75.03, \mathrm{SEM}=0.96)$ than at baseline (mean $=46.45$, SEM $=1.00$ ) across groups (Fig. $2 b$ ). We also observed a significant main effect of the group $\left(F_{1,753}=4.2, p=0.040\right)$ on self-reported expectations, with TMD patients displaying overall greater expectations (mean $=62.32$, $\mathrm{SEM}=1.12)$ than controls $($ mean $=59.16, \mathrm{SEM}=1.06$ ), with a nonsignificant interaction between group and time $\left(F_{1,753}=0.15, p=0.696\right)$. In the TMD cohort, both baseline $(r=0.12, p=0.018)$ and reinforced $(r=0.161, p=$ 0.002 ) expectations were positively associated with placebo hypoalgesia. On the contrary, neither baseline ( $r=$ $0.06, p=0.213)$ nor reinforced $(r=0.06, p=0.261)$ expectations showed significant correlations with placebo effects in the healthy control cohort.

We therefore explored the potential mediation role of reinforced expectations in driving placebo effects in TMD patients and controls, respectively. Reinforced expectations did not mediate the placebo effects in either TMD patients (path $\mathrm{b}=0.05, p=0.219$ ) or controls (path $\mathrm{b}=$ 


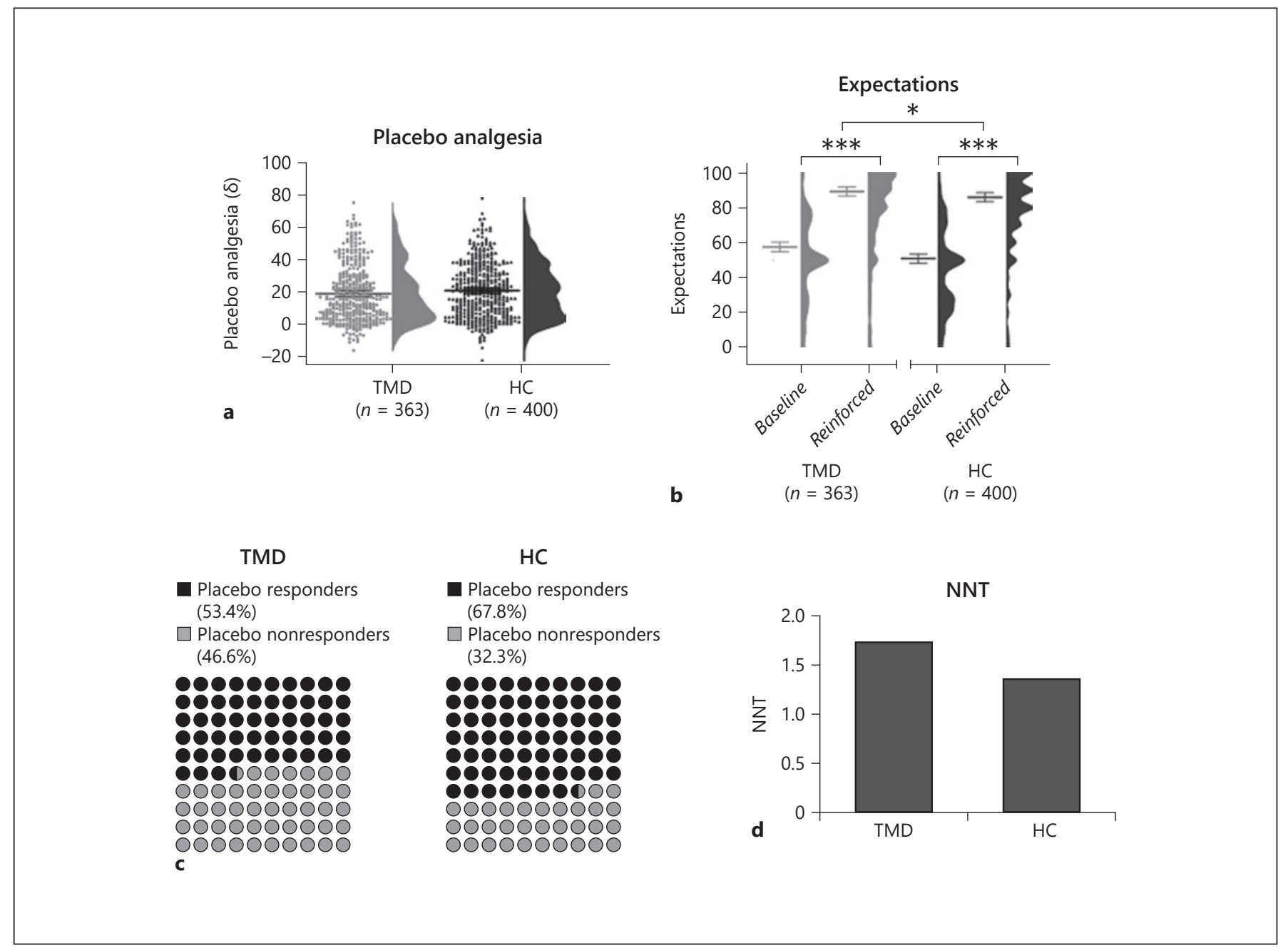

Fig. 2. a-d Expectations, placebo effects, proportion of responders, and NNT. There was no difference in TMD vs. control participants in terms of the magnitude of placebo analgesia. The average of the 6 red-minus-green $\delta$ scores of pain intensity ratings from each individual are presented. Each point represents individual placebo effects. Mean $\pm 95 \%$ CI along with frequency distributions (half violin plots on the right) are shown. Self-reported expectations before and after the conditioning phase for both TMD and control participants are shown. The reinforced expectation ratings after the conditioning phase were significantly higher than baseline expectations in both TMD patients and healthy controls. TMD patients had overall greater pain relief expectations than did the healthy controls. Data are presented as means $\pm 95 \% \mathrm{CI}$ along with the frequency distribution (half violin plots on the right). c Each study participant was classified based on a permutation test as a "responder." The null hypothesis was generated by randomly resampling 1,000 times the distribution of pain ratings, which provides a large set of possible $t$ values obtained from the rearrangement of the VAS pain ratings. The overall $t$ value obtained between red- and green-related pain reports was used to determine whether the null hypothesis could be rejected $(p<0.05)$. In the cases where the null hypothesis could not be rejected, the study participant would be stratified as a "nonresponder." Alternatively, the participant would be stratified as a "responder" if there was a significant diminution in the pain ratings. This permutation-based approach offers the advantage of accounting for the variability across pain ratings during the trial-by-trial reports during the placebo test phase. The proportion of placebo responders within TMD patients (53.4\%) was significantly lower $(p<0.001)$ than the proportion of responders in control participants $(67.8 \%)$. $\mathbf{d}$ The NNT refers to the number of patients we would need to treat to prevent an unwanted additional adverse event. For example, if a drug has an NNT of 100 , it means that you have to treat 100 people with that drug to prevent an additional person from experiencing the unwanted adverse event. The NNT for TMD patients was larger than the NNT for control participants, indicating that fewer TMD patients would benefit from placebo analgesia compared to controls. $\mathrm{HC}$, healthy controls. ${ }^{*} p=0.05$; $^{* * *} p<0.001$. 
$0.01, p=0.747)$; however, the strength of conditioning (i.e., larger differences between red and green stimulations during the conditioning phase) was associated with greater reinforced expectations (TMD patients: path $\mathrm{a}=$ $0.54, p<0.001$; healthy controls: path a $=0.38, p<0.001$; online suppl. Fig. S1A, B) and mediated larger placebo analgesia (TMD patients: path $c=0.24, p<0.001$; healthy controls: path $c=0.17, p=0.001$ ).

In terms of placebo responsiveness, we identified $53.4 \%$ of the TMD patients as placebo responders, which was significantly lower than in the healthy control participants, where $67.8 \%$ of the cohort were responders $\left(\chi^{2}=16.36, p<0.001\right.$; Fig. $\left.2 c\right)$. Importantly, similar NNT were observed in the TMD (NNT $=1.88)$ and healthy control $(\mathrm{NNT}=1.47)$ cohorts $(Z=1.07, p=0.143$; Fig. $2 \mathrm{~d})$.

\section{Discussion}

This is the largest study to date comparing the occurrence of placebo effects in chronic pain participants and healthy controls, testing the role of prior therapeutic experiences and expectations in driving such effects. Chronic pain participants displayed higher expectations of relief but identical magnitudes of placebo effects when compared with healthy controls. While expectations did not mediate placebo effects, prior perception of hypoalgesia did. Specifically, participants' therapeutic experiences during the conditioning phase were associated with larger reinforced (postconditioning) expectations and subsequent larger placebo effects.

These findings are consistent with previous studies [39-42]. In fact, no difference in placebo analgesia responsiveness was reported between healthy controls and patients with low back pain [43], episodic migraine [44], irritable bowel syndrome [45], and atopic dermatitis patients [46]. Rating expectations has been suggested as a potential tool for predicting placebo responses [39]. Contrary to this, despite the larger reinforced expectation levels that TMD patients reported and the significant association with placebo effects, expectations did not mediate or predict placebo responsiveness. Rather, prior experiences of pain reduction during the conditioning phase directly mediated subsequent placebo effects. This finding echoes previous ones outlining that various degrees of exposure to analgesic benefits such as the length of training [47], prior experience with either an effective or an ineffective treatment [30], the schedule (1 day apart vs. 1 week apart) [48], and the conditioning paradigm (continuous vs. partial rein- forcement) [29] influence the occurrence and magnitude of (learned) placebo effects. In the current study, prior therapeutic experiences and learning triggered placebo responsiveness in both chronic pain participants and healthy controls, suggesting that these factors can be used to engage the placebo effects (i.e., endogenous opioid and nonopioid systems) [49] regardless of chronic pain status.

When considering the response rate, we observed that the prevalence of responders was significantly larger in the controls than in TMD patients, with 67.8 versus $53.4 \%$ of placebo responders, respectively. Given that these results can be informative for randomized clinical trials [50], we computed the NNT for the cases and controls and found a similar NNT for TMD patients and controls, outlining that the placebo effect rate and magnitude can still be significant in patients despite the presence of chronic pain. Moreover, the NNT of 1.88 observed in TMD is comparable to clinical chronic pain pharmacology (e.g., anticonvulsants and opioids), which ranges from an NNT of 1.7 to 3 [51].

Despite the novelty of these findings that indicate that chronic pain participants not only respond to placebos but also respond with a comparable magnitude to painfree controls, there are some limitations to be acknowledged. First, although expectation ratings were larger in the TMD patients than in the controls, they did not predict placebo effects, contrary to prior studies [for a review, see 39] and this may have depended on the experimental setting. Second, we did not include a group with no conditioning [for an example, see 19], limiting the generalizability of these results to verbally induced placebo effects. Third, we used conditioning strengths to simulate prior therapeutic experiences similarly to a previous study [52]. This may differ from clinical medical histories, and further research is needed to develop behavioral tools to accurately predict placebo effects. Beyond these limitations, this study indicates that health providers should account for prior therapeutic experiences that might affect individuals' response to placebos, or their likelihood of benefiting from placebo mechanisms with promising applications for medicine and drug development.

\section{Acknowledgement}

The authors thank all participants for taking their precious time to participate in this research project. We acknowledge Cynthia Renn, Susan Dorsey, and Joel Greenspan for their continuous support during the execution of this project. 


\section{Statement of Ethics}

This study was approved by the University of Maryland Institutional Review Board (HP-00068315) and it was conducted in accordance with the World Medical Association Declaration of Helsinki. All of the participants provided verbal and written consent to participate.

\section{Disclosure Statement}

The authors have no conflict of interests to declare.

\section{Funding Sources}

This research was supported by National Institute Dental Craniofacial Research (NIDCR; R01 DE025946 to Luana Colloca). The funding agencies have no role in this study. The views expressed here are the authors' own and do not reflect the position or policy of the National Institutes of Health or any other part of the federal government.

\section{Author Contributions}

Luana Colloca designed this study, contributed to data collection and analyses, interpreted the results, and drafted this paper. Titilola Akintola, Nathaniel R. Haycock, Nicole Corsi, Maxie Blasini, and Lieven A. Schenk collected the data. Jane Phillips screened and confirmed the diagnosis of TMD. Luana Colloca and Sharon Thomas screened the healthy controls. Yang Wang analyzed the data in collaboration with Luana Colloca. All of the authors commented on and approved the final draft.

\section{References}

1 Evers AW, Colloca L, Blease C, Annoni M, Atlas LY, Benedetti F, et al. Implications of Placebo and Nocebo Effects for Clinical Practice: expert Consensus. Psychother Psychosom. 2018;87(4):204-10.

2 Fava GA, Guidi J, Rafanelli C, Rickels K. The Clinical Inadequacy of the Placebo Model and the Development of an Alternative Conceptual Framework. Psychother Psychosom. 2017;86(6):332-40

3 Colloca L, Barsky AJ. Placebo and Nocebo Effects. N Engl J Med. 2020 Feb;382(6):554-61.

4 Peerdeman KJ, van Laarhoven AI, Keij SM, Vase L, Rovers MM, Peters ML, et al. Relieving patients' pain with expectation interventions: a meta-analysis. Pain. 2016 Jun; 157(6): 1179-91.

5 Scrivani SJ, Keith DA, Kaban LB. Temporomandibular disorders. N Engl J Med. 2008 Dec;359(25):2693-705.

6 Dworkin SF, Huggins KH, LeResche L, Von Korff M, Howard J, Truelove E, et al. Epidemiology of signs and symptoms in temporomandibular disorders: clinical signs in cases and controls. J Am Dent Assoc. 1990 Mar; 120(3):273-81.

7 Dworkin SF, Massoth DL. Temporomandibular disorders and chronic pain: disease or illness? J Prosthet Dent. 1994 Jul;72(1):29-38.

8 Fricton J. Temporomandibular disorders: a human systems approach. J Calif Dent Assoc. 2014;42(8):523-33.

9 Bender SD. Orofacial pain and headache: a review and look at the commonalities. Curr Pain Headache Rep. 2014 Mar; 18(3):400.

10 Murphy MK, MacBarb RF, Wong ME, Athanasiou KA. Temporomandibular disorders: a review of etiology, clinical management, and tissue engineering strategies. Int J Oral Maxillofac Implants. 2013 Nov-Dec;28(6):e393414

Placebo Effects and Chronic Orofacial Pain
11 Vase L, Robinson ME, Verne GN, Price DD. The contributions of suggestion, desire, and expectation to placebo effects in irritable bowel syndrome patients. An empirical investigation. Pain. 2003 Sep;105(1-2):17-25.

12 Kaptchuk TJ, Kelley JM, Conboy LA, Davis $\mathrm{RB}$, Kerr CE, Jacobson EE, et al. Components of placebo effect: randomised controlled trial in patients with irritable bowel syndrome. BMJ. 2008 May;336(7651):999-1003.

13 Vase L, Robinson ME, Verne GN, Price DD. Increased placebo analgesia over time in irritable bowel syndrome (IBS) patients is associated with desire and expectation but not endogenous opioid mechanisms. Pain. 2005 Jun;115(3):338-47.

14 Vase L, Petersen GL, Lund K. Placebo effects in idiopathic and neuropathic pain conditions. Handb Exp Pharmacol. 2014;225:12136.

15 Petersen GL, Finnerup NB, Grosen K, Pilegaard HK, Tracey I, Benedetti F, et al. Expectations and positive emotional feelings accompany reductions in ongoing and evoked neuropathic pain following placebo interventions. Pain. 2014 Dec;155(12):2687-98.

16 Petersen GL, Finnerup NB, Nørskov KN, Grosen K, Pilegaard HK, Benedetti F, et al. Placebo manipulations reduce hyperalgesia in neuropathic pain. Pain. 2012 Jun;153(6): 1292-300.

17 Hashmi JA, Baria AT, Baliki MN, Huang L, Schnitzer TJ, Apkarian AV. Brain networks predicting placebo analgesia in a clinical trial for chronic back pain. Pain. 2012 Dec;153(12): 2393-402.

18 Carvalho C, Caetano JM, Cunha L, Rebouta P, Kaptchuk TJ, Kirsch I. Open-label placebo treatment in chronic low back pain: a randomized controlled trial. Pain. 2016 Dec; 157(12):2766-72.
19 Schmitz J, Müller M, Stork J, Eichler I, Zöllner C, Flor H, et al. Positive Treatment Expectancies Reduce Clinical Pain and Perceived Limitations in Movement Ability Despite Increased Experimental Pain: A Randomized Controlled Trial on Sham Opioid Infusion in Patients with Chronic Back Pain. Psychother Psychosom. 2019;88(4):203-14.

20 Kam-Hansen S, Jakubowski M, Kelley JM, Kirsch I, Hoaglin DC, Kaptchuk TJ, et al. Altered placebo and drug labeling changes the outcome of episodic migraine attacks. Sci Transl Med. 2014 Jan;6(218):218ra5.

21 Tétreault P, Mansour A, Vachon-Presseau E, Schnitzer TJ, Apkarian AV, Baliki MN. Brain connectivity predicts placebo response across chronic pain clinical trials. PLoS Biol. 2016 Oct;14(10):e1002570.

22 Forsberg JT, Martinussen M, Flaten MA. The Placebo Analgesic Effect in Healthy Individuals and Patients: A Meta-Analysis. Psychosom Med. 2017 May;79(4):388-94.

23 Schiffman E, Ohrbach R, Truelove E, Look J Anderson G, Goulet JP, et al.; International RDC/TMD Consortium Network, International association for Dental Research; Orofacial Pain Special Interest Group, International Association for the Study of Pain. Diagnostic Criteria for Temporomandibular Disorders (DC/TMD) for Clinical and Research Applications: recommendations of the International RDC/TMD Consortium Network* and Orofacial Pain Special Interest Group. J Oral Facial Pain Headache. 2014; 28(1):6-27.

24 Zakrzewska JM. Differential diagnosis of facial pain and guidelines for management. $\mathrm{Br} \mathrm{J}$ Anaesth. 2013 Jul;111(1):95-104.

25 Dixon D, Pollard B, Johnston M. What does the chronic pain grade questionnaire measure? Pain. 2007 Aug;130(3):249-53. 
26 Ohrbach R, Larsson P, List T. The jaw functional limitation scale: development, reliability, and validity of 8 -item and 20 -item versions. J Orofac Pain. 2008;22(3):219-30.

27 Ohrbach R, Markiewicz MR, McCall WD Jr. Waking-state oral parafunctional behaviors: specificity and validity as assessed by electromyography. Eur J Oral Sci. 2008 Oct;116(5): 438-44.

28 Schafer SM, Colloca L, Wager TD. Conditioned placebo analgesia persists when subjects know they are receiving a placebo. J Pain. 2015 May; 16(5):412-20.

29 Au Yeung ST, Colagiuri B, Lovibond PF, Colloca L. Partial reinforcement, extinction, and placebo analgesia. Pain. 2014 Jun;155(6): $1110-7$.

30 Colloca L, Benedetti F. How prior experience shapes placebo analgesia. Pain. 2006 Sep; 124(1-2):126-33.

31 Colloca L, Benedetti F. Placebo analgesia induced by social observational learning. Pain. 2009 Jul;144(1-2):28-34.

32 Colloca L, Sigaudo M, Benedetti F. The role of learning in nocebo and placebo effects. Pain. 2008 May; 136(1-2):211-8.

33 Colloca L, Tinazzi M, Recchia S, Le Pera D, Fiaschi A, Benedetti F, et al. Learning potentiates neurophysiological and behavioral placebo analgesic responses. Pain. 2008 Oct; 139(2):306-14.

34 Lui F, Colloca L, Duzzi D, Anchisi D, Benedetti F, Porro CA. Neural bases of conditioned placebo analgesia. Pain. 2010 Dec 151(3):816-24.
35 Colloca L, Wang Y, Martinez PE, Chang YC, Ryan KA, Hodgkinson C, et al. OPRM1 rs1799971, COMT rs4680, and FAAH rs324420 genes interact with placebo procedures to induce hypoalgesia. Pain. 2019 Aug; 160(8):1824-34.

36 Furukawa TA, Leucht S. How to obtain NNT from Cohen's d: comparison of two methods. PLoS One. 2011 Apr;6(4):e19070.

37 Hayes AF, Preacher KJ. Quantifying and Testing Indirect Effects in Simple Mediation Models When the Constituent Paths Are Nonlinear. Multivariate Behav Res. 2010 Aug; 45(4):627-60

38 Hayes AF. Beyond Baron and Kenny: statistical mediation analysis in the new millennium. Commun Monogr. 2009;76(4):408-20.

39 Vase L, Amanzio M, Price DD. Nocebo vs. placebo: the challenges of trial design in analgesia research. Clin Pharmacol Ther. 2015 Feb;97(2):143-50.

40 Vase L, Vollert J, Finnerup NB, Miao X, Atkinson G, Marshall S, et al. Predictors of the placebo analgesia response in randomized controlled trials of chronic pain: a meta-analysis of the individual data from nine industrially sponsored trials. Pain. 2015 Sep;156(9): 1795-802.

41 Colloca L. Placebo, nocebo, and learning mechanisms. Handb Exp Pharmacol. 2014; 225:17-35.

42 Colloca L, Miller FG. Role of expectations in health. Curr Opin Psychiatry. 2011 Mar; 24(2):149-55.

43 Charron J, Rainville P, Marchand S. Direct comparison of placebo effects on clinical and experimental pain. Clin J Pain. 2006 Feb; 22(2):204-11.
44 Linnman C, Catana C, Petkov MP, Chonde DB, Becerra L, Hooker J, et al. Molecular and functional PET-fMRI measures of placebo analgesia in episodic migraine: preliminary findings. Neuroimage Clin. 2017 Nov; 17:680-90.

45 Lee HF, Hsieh JC, Lu CL, Yeh TC, Tu CH, Cheng CM, et al. Enhanced affect/cognitionrelated brain responses during visceral placebo analgesia in irritable bowel syndrome patients. Pain. 2012 Jun;153(6):1301-10.

46 Klinger R, Soost S, Flor H, Worm M. Classical conditioning and expectancy in placebo hypoalgesia: a randomized controlled study in patients with atopic dermatitis and persons with healthy skin. Pain. 2007 Mar;128(1-2):31-9.

47 Colloca L, Petrovic P, Wager TD, Ingvar M, Benedetti F. How the number of learning trials affects placebo and nocebo responses. Pain. 2010 Nov;151(2):430-9.

48 Benedetti F, Pollo A, Colloca L. Opioid-mediated placebo responses boost pain endurance and physical performance: is it doping in sport competitions? J Neurosci. 2007 Oct; 27(44):11934-9.

49 Colloca L. The Placebo Effect in Pain Therapies. Annu Rev Pharmacol Toxicol. 2019 Jan; 59(1):191-211.

50 Katz N, Paillard FC, Van Inwegen R. A review of the use of the number needed to treat to evaluate the efficacy of analgesics. J Pain. 2015 Feb;16(2):116-23.

51 Lynch ME, Watson CP. The pharmacotherapy of chronic pain: a review. Pain Res Manag. 2006;11(1):11-38.

52 Kessner S, Wiech K, Forkmann K, Ploner M, Bingel U. The effect of treatment history on therapeutic outcome: an experimental approach. JAMA Intern Med. 2013 Aug;173(15):1468-9. 